\title{
The Core Technology Research of GPS Navigation System Synchronization
}

\author{
Haiyi Sun ${ }^{1, a}$, Ning $\mathrm{Li}^{2, b^{*}}$, Zhongtang Chen ${ }^{1, c}$ and Shuxian Miao ${ }^{1, d}$ \\ ${ }^{1}$ College of Science, Shenyang JianZhu University, Shenyang, China \\ ${ }^{2}$ College of Sciences, Northeastern University, Shenyang, China \\ ashy_xx@163.com, ${ }^{b}$ lining80@163.com, ${ }^{\circ}$ chen630609@163.com, ${ }^{d}$ miaoshuxian@163.com
}

Keywords: GPS navigation system; Coupling delays; Delayed nodes; Adaptive control; Lag synchronization

\begin{abstract}
The clock synchronization of GPS navigation system is a key technology. This paper is based on GPS clock system, aiming at the asynchronous problem of GPS in the clock, and establishes a more realistic nonlinear dynamic model with coupling delays and delays in the dynamical nodes than before. The clock lag synchronization criteria for the model have been studied. Via the theory of Lyapunov stability, adaptive control technique and delay control methods, we design the adaptive lag feedback synchronization controller. It should be pointed out that the node dynamic need not satisfy the very strong and the coupling matrices are not assumed to be symmetric. The accuracy, real-time, stable and reliable clock data is acquired. Finally, the numerical example of the adaptive lag synchronization problem has been also provided to demonstrate the effectiveness of the theory.
\end{abstract}

\section{Introduction}

GPS is a precise new-generation location system promoted by the fast development of the modem science and technology. It has many characteristics such as all global all-weather, high position accuracy continuous and real-time. It has been widely developed in the field of military and civil affairs. In these years, the technology of GPS location develops very quickly, the technology of location and navigation comes into a new stage, the clock synchronization of GPS navigation system is a key technology. To have high precision and stability, the random error of the satellite clock is needed to be corrected, and achieving the synchronization of the receiver clock and GPS clock. Establish the mathematical model, so that the deviation can be accepted [1-3].

Recently, the study of various complex networks has attracted increasing attentions from researchers in various fields of physics, mathematics, engineering, biology, and sociology [4, 5]. There have been a rich body of literature on analyzing complex networks, and one of the most significant dynamical behaviors of complex networks that have been widely investigated is the synchronization motion of its dynamical elements [6-8]. Some synchronization phenomena are very useful for us, such as the synchronous transfer of digital or analog signals in communication networks [5].

In practice, the information transmission within complex networks is in general not instantaneous since the signal traveling speed is limited, and this is very common in biological and physical networks. This fact gives rise to the time delays that may cause undesirable dynamic network behaviors such as oscillation and instability. Therefore, time delays should be modeled in order to simulating more realistic networks. The lag synchronization of GPS clock system, which is considered both coupling delays and delays in the dynamical nodes, has only been lightly covered [8-11]. As a matter of fact, we can find a lot of examples in the real world which are characterized by complex dynamical networks with coupling delays and delays in the dynamical nodes. Therefore, it is imperative to further investigate complex dynamical networks with coupling delays and delays in the dynamical nodes. 
In this paper, we first introduce a more general model of GPS clock system with coupling delays and delays in the dynamical nodes than before. Then we further study the adaptive lag synchronization of this model. Based on the theory of asymptotic stability of time-delay systems and Lyapunov method combined with adaptive control technique, we treat the realistic case of adaptive lag feedback synchronization controller of the networks. It should be pointed out that the node dynamic need not satisfy the very strong and the coupling matrices are not assumed to be symmetric.

\section{Model Description and Preliminaries}

The control GPS clock system with coupling delays and delays in the dynamical nodes can be described as follows:

$$
\dot{x}_{i}(t)=f\left(x_{i}(t), x_{i}\left(t-\tau_{1}\right)\right)+\sum_{j=1}^{N} a_{i j} \Gamma_{1} x_{j}(t)+\sum_{j=1}^{N} b_{i j} \Gamma_{2} x_{j}\left(t-\tau_{2}\right)+u_{i}(t),
$$

where $x_{i}(t)=\left(x_{i 1}, x_{i 2}, \cdots, x_{i n}\right)^{\mathrm{T}} \in R^{n}$ is the state and input variable of GPS node $i$ at time $t . i=1,2, \cdots, N$ $f: R^{n} \times R^{n} \rightarrow R^{n}$ is a continuous and differentiable function, $\tau_{1}$ and $\tau_{2}$ are the time delay of coupling delays and delays in the dynamical nodes, respectively, which are arbitrary but bounded, i.e., $\tau_{1}, \tau_{2} \in(0, h]$, where $h$ is a positive constant. $A=\left(a_{i j}\right) \in R^{N \times N}$ and $B=\left(b_{i j}\right) \in R^{N \times N}$ are the coupling matrices with zero-sum rows, which represent the coupling strength and the underlying topology for non-delayed configuration and delayed one $\tau_{2}$ at time $t$, respectively, $a_{i j} \geq 0, b_{i j} \geq 0$ for $i \neq j$, $a_{i j}, b_{i j}$ are defined as follows: if there is a connection from node $j$ to node $i(i \neq j) a_{i j}>0, b_{i j}>0$, otherwise $a_{i j}=0, b_{i j}=0(i \neq j)$, and $\Gamma_{1}, \Gamma_{2} \in R^{n \times n}$ are positive diagonal matrices which describe the individual couplings between node $i$ and $j$ for non-delayed configuration and delayed one $\tau_{2}$ at time $t$ respectively.

When the GPS clock system (1) achieves synchronization, namely, the states $x_{1}(t) \rightarrow \cdots \rightarrow x_{N}(t)$ $\rightarrow s(t)$, as $t \rightarrow \infty$, where $s(t) \in R^{n}$ is a solution of a satellite clock system, i.e.

$$
s(t)=f\left(s(t), s\left(t-\tau_{1}\right)\right) .
$$

$s(t)$ Can be an equilibrium point, a nontrivial periodic orbit, or even a chaotic orbit. Let $C\left([-h, 0], R^{n}\right)$ be the Banach space of continuous functions mapping the interval $[-h, 0]$ into $R^{n}$ with the norm $\|\phi\|=\sup _{-h \leq \theta \leq 0}\|\phi(\theta)\|$, where $\|\cdot\|$ is the Euclidean norm. The rigorous mathematical definition of synchronization for GPS clock system (1) is introduced as follows.

Definition 1. Let $x_{i}\left(t ; t_{0} ; \phi\right), i=1,2, \cdots, N$ be a solution of GPS clock system (1) and satellite (2), where $\phi=\left(\phi_{1}^{\mathrm{T}}, \phi_{2}^{\mathrm{T}}, \cdots, \phi_{N}^{\mathrm{T}}\right)^{\mathrm{T}}, \quad \phi_{i}=\phi_{i}(\theta) \in C \quad\left(\left[-\tau_{m}, 0\right], R^{n}\right)$ are initial conditions. If there is a nonempty subset $\Lambda \subseteq R^{n}$, such that $\phi_{i}$ take values in $\Lambda$ and $x_{i}\left(t ; t_{0} ; \phi\right) \in R^{n}$ for all $t \geq t_{0}$ and

$$
\lim _{t \rightarrow \infty}\left\|x_{i}\left(t ; t_{0} ; \phi\right)-s\left(t-\sigma ; t_{0} ; s_{0}\right)\right\|=0, \quad i=1,2, \cdots, N
$$

where $s\left(t ; t_{0} ; s_{0}\right)$ is a solution of the system (2), $\sigma$ is the delay of lag synchronization, then the GPS clock system (1) is said to realize synchronization, and $\Lambda \times \Lambda \times \cdots \times \Lambda$ is called the region of synchrony of the GPS clock system (1).

Define the error vector by

$$
e_{i}(t)=x_{i}(t)-s(t-\sigma), \quad i=1,2, \cdots, N
$$

Then the error system can be described by 


$$
\dot{e}_{i}(t)=f\left(x_{i}(t), x_{i}\left(t-\tau_{1}\right)\right)-f\left(s(t-\sigma), s\left(t-\tau_{1}-\sigma\right)\right)+\sum_{j=1}^{N} a_{i j} \Gamma_{1} e_{j}(t)+\sum_{j=1}^{N} b_{i j} \Gamma_{2} e_{j}\left(t-\tau_{2}\right)+u_{i}(t),
$$

Then the synchronization problem of the GPS clock system (1) is equivalent to the problem of stabilization of the error dynamical system (5).

Assumption 1. We always assume that $f(\bullet)$ satisfied the uniform Lipschiz condition with respect to the time $t$, i.e., for any $x(t)=\left(x_{1}(t), x_{2}(t), \cdots, x_{n}(t)\right)^{\mathrm{T}}, y(t)=\left(y_{1}(t), y_{2}(t), \cdots, y_{n}(t)\right)^{\mathrm{T}} \in R^{n}$, there exist constants $1 \leq i \leq n, k_{i j}>0$ satisfying

$$
\left|f_{i}\left(x(t), x\left(t-\tau_{1}\right)\right)-f_{i}\left(y(t), y\left(t-\tau_{1}\right)\right)\right| \leq \sum_{j=1}^{n} k_{i j}\left(\left|x_{j}(t)-y_{j}(t)\right|+\left|x_{j}\left(t-\tau_{1}\right)-y_{j}\left(t-\tau_{1}\right)\right|\right) .
$$

\section{Adaptive Pinning Synchronization Control of the GPS Clock System}

In this section, we are in the position to present our main results for lag synchronization control of the GPS clock system with coupling delays and delays in the dynamical nodes by adaptive feedback controllers. In the following, assume that $\|A\|_{2}=\alpha$ and denote by $\rho_{\min }$ the minimum eigenvalue of the matrix $\left(\bar{A}+\bar{A}^{\mathrm{T}}\right) / 2$. Let $\bar{A}^{S}=\left(\bar{A}+\bar{A}^{\mathrm{T}}\right) / 2$, where $\bar{A}$ is a modified matrix of $A$ via replacing the diagonal elements $a_{i i}$ by $\left(\rho_{\min } / \alpha\right) a_{i i} . \bar{B}$ has the same meaning of $\bar{A} \cdot p_{i}=\frac{1}{2} \sum_{s=1}^{m}\left(2 k_{i s}^{2 \varepsilon}+k_{s i}^{2(1-\varepsilon)}\right)$, $q_{i}=\sum_{s=1}^{m} k_{s i}^{2(1-\varepsilon)}, \quad p=\max _{1 \leq i \leq m} p_{i}, \quad q=\max _{1 \leq i \leq m} q_{i},\left\|\Gamma_{i}\right\|=\gamma_{i}$.

Theorem 1. Suppose Assumption 1 holds. Then the controlled GPS clock system (1) is globally lag synchronized with adaptive controllers

$$
u_{i}(t)=\left\{\begin{array}{cc}
-\alpha_{i}(t)\left(x_{i}(t)-s(t-\sigma)\right), & 1 \leq i \leq h ; \\
0, & h+1 \leq i \leq N ;
\end{array}\right.
$$

and updating laws

$$
\dot{\alpha}_{i}(t)=\beta_{i}\left\|x_{i}(t)-s(t-\sigma)\right\|^{2}
$$

where $\beta_{i}>0(i=1,2, \cdots, h)$.

Proof: Selecting a Lyapunov-Krasovskii function of the form

$$
V(t)=\frac{1}{2} \sum_{i=1}^{N} e_{i}^{\mathrm{T}}(t) e_{i}(t)+\sum_{i=1}^{N} \frac{q}{2} \int_{t-\tau_{1}}^{t} e_{i}^{\mathrm{T}}(s) e_{i}(s) \mathrm{d} s+\sum_{i=1}^{N} \mu \int_{t-\tau_{2}}^{t} e_{i}^{\mathrm{T}}(s) e_{i}(s) \mathrm{d} s+\sum_{i=1}^{N} \frac{1}{2 \beta}\left(\alpha_{i}(t)-\alpha\right)^{2} .
$$

Combining the Assumption 1, the time derivative of each $V(t)$ along the trajectories of the error complex dynamical networks (5) can be processed as

$$
\begin{aligned}
\dot{V}(t)= & \sum_{i=1}^{N} e_{i}^{\mathrm{T}}(t)\left[f\left(x_{i}(t), x_{i}\left(t-\tau_{1}\right)\right)-f\left(s(t), s\left(t-\tau_{1}\right)\right)+\sum_{j=1}^{N} a_{i j} \Gamma_{1} e_{j}(t)+\sum_{j=1}^{N} b_{i j} \Gamma_{2} e_{j}\left(t-\tau_{2}\right)\right] \\
& -\sum_{i=1}^{h} \alpha_{i}(t) e_{i}^{\mathrm{T}}(t) e_{i}(t)+\sum_{i=1}^{N} \frac{q}{2}\left[e_{i}^{\mathrm{T}}(t) e_{i}(t)-e_{i}^{\mathrm{T}}\left(t-\tau_{1}\right) e_{i}\left(t-\tau_{1}\right)\right] \\
& +\sum_{i=1}^{N} \mu\left[e_{i}^{\mathrm{T}}(t) e_{i}(t)-e_{i}^{\mathrm{T}}\left(t-\tau_{2}\right) e_{i}\left(t-\tau_{2}\right)\right]+\sum_{i=1}^{h}\left[\alpha_{i}(t)-\alpha\right] e_{i}^{\mathrm{T}}(t) e_{i}(t) \\
\leq & p \sum_{i=1}^{N} e_{i}^{\mathrm{T}}(t) e_{i}(t)+\frac{1}{2} \sum_{i=1}^{N} e_{i}^{\mathrm{T}}\left(t-\tau_{1}\right) e_{i}\left(t-\tau_{1}\right)+\sum_{i=1}^{N} a_{i i} \gamma_{1(\min )}\left\|e_{i}(t)\right\|^{2}+\sum_{i=1}^{N} \sum_{j=1, j \neq i}^{N} a_{i j} \gamma_{1}\left\|e_{i}(t)\right\|\left\|e_{j}(t)\right\| \\
& +\sum_{i=1}^{N} \sum_{j=1, j \neq i}^{N} b_{i j} \gamma_{2}\left\|e_{i}(t)\right\|\left\|e_{j}\left(t-\tau_{2}\right)\right\|+\sum_{i=1}^{N} b_{i i} \gamma_{2(\min )}\left\|e_{i}(t)\right\|\left\|e_{i}\left(t-\tau_{2}\right)\right\|+\sum_{i=1}^{N} \frac{q}{2}\left\|e_{i}(t)\right\|^{2}-\sum_{i=1}^{N} \frac{q}{2}\left\|e_{i}\left(t-\tau_{1}\right)\right\|^{2}
\end{aligned}
$$




$$
\begin{aligned}
& +\sum_{i=1}^{N} \mu\left\|e_{i}(t)\right\|^{2}-\sum_{i=1}^{N} \mu\left\|e_{i}\left(t-\tau_{2}\right)\right\|^{2}-\sum_{i=1}^{h} \alpha\left\|e_{i}(t)\right\|^{2} \\
= & p \bar{e}^{\mathrm{T}}(t) \bar{e}(t)+\bar{e}^{\mathrm{T}}(t) \gamma_{1} \bar{A}^{S} \bar{e}(t)+\bar{e}^{\mathrm{T}}(t) \gamma_{2} \bar{B}^{S} \bar{e}\left(t-\tau_{2}\right)+\mu \bar{e}^{\mathrm{T}}(t) \bar{e}(t)+\frac{1}{2} q \bar{e}^{\mathrm{T}}(t) \bar{e}(t) \\
& -\mu \bar{e}^{\mathrm{T}}\left(t-\tau_{2}\right) \bar{e}\left(t-\tau_{2}\right)-\bar{e}^{\mathrm{T}}(t) D \bar{e}(t) \\
= & \overline{\bar{e}}^{\mathrm{T}}\left(t, t-\tau_{1}, t-\tau_{2}\right) \Phi \overline{\bar{e}}\left(t, t-\tau_{1}, t-\tau_{2}\right),
\end{aligned}
$$

where

$$
\overrightarrow{\bar{e}}\left(t, t-\tau_{1}, t-\tau_{2}\right)=\left(\bar{e}^{\mathrm{T}}(t), \bar{e}^{\mathrm{T}}\left(t-\tau_{1}\right), \bar{e}^{\mathrm{T}}\left(t-\tau_{2}\right)\right)^{\mathrm{T}}, \Phi=\left[\begin{array}{cc}
\left(p+\mu+\frac{q}{2}\right) I_{N}+\gamma_{1} \bar{A}^{S}-D & \frac{\gamma_{2}}{2} \bar{B}^{s} \\
* & -\mu I_{N}
\end{array}\right],
$$

Due to the fact that (9), one has $\left(p+\mu+\frac{q}{2}\right) I_{N}+\gamma_{1} \bar{A}^{S}-D+\frac{\gamma_{2}}{4 \mu} \bar{B}^{S^{2}}<0$ when $\beta_{i}(i=1,2, \cdots, h)$ are large enough, i.e., $\Phi$ is negative definite. We have $\dot{V}(t)<0$. Then the GPS clock system (1) is globally lag synchronized. Theorem 1 is proved.

\section{Numerical Simulation}

In this section, the simulation example is given to verify the criteria established above. Here, consider the GPS clock system (1) with $\tau_{1}=0.5, \tau_{2}=1, \sigma=2$ that consists of 10 identical Lorenz systems adaptive controller. We know the Lorenz system can be mathematically described as

$$
f\left(x_{i}(t), t\right)=\left\{\begin{array}{l}
10\left(x_{i 2}-x_{i 1}\right) \\
28 x_{i 1}-x_{i 1} x_{i 3}-x_{i 2}, \\
x_{i 1} x_{i 2}-\frac{8}{3} x_{i 3} .
\end{array}\right.
$$

In the simulation, the asymmetric coupling matrices as $A$ and are random and satisfied with the coupling condition. And from [10], we know the Lorenz system satisfies Assumption 1. In this section, the parameters are set as $\beta_{i}=1$, else. The time series of the GPS clock system (1) with adaptive controllers are numerically demonstrated as the figure 1 . Clear, all errors are rapidly achieving synchronization. For this simulation, the initial values of states $x_{i}(0)(i=1,2, \cdots, 10)$ are random and $s(0)=(1,1,1)^{\mathrm{T}}$.
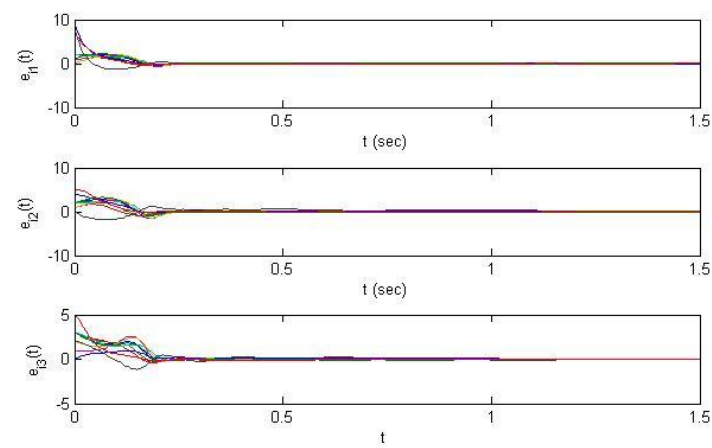

Figure 1. Synchronization errors of GPS clock system (1) under the adaptive pinning controller (7)-(8)

\section{Conclusion}

A general GPS clock system with coupling delays and delays in the dynamical nodes has been studied in this paper. We design the adaptive lag feedback synchronization controllers via the theory of 
Lyapunov stability, adaptive technique and delay control theory. The controllers are very useful for understanding the lag synchronization in GPS clock system with coupling delays and delays in the dynamical nodes. Moreover, the resulting adaptive feedback controllers for achieving lag synchronization are expressed in simple form that can be readily applied in practical situations. Finally, the effectiveness of these synchronization controllers is verified by numerical simulations.

\section{Acknowledgment}

This work was partially supported by the Fundamental Research Funds for the Central Universities (N140504009), the Science Foundation of Ministry of Housing and Urban-Rural Development (grant no. K82016019), the Discipline HanYu Project of Shenyang Jianzhu University (grant no. XKHY-79) and the 9th group of education scientific research project topics of Shenyang Jianzhu University (grant no.20160124).

\section{References}

[1] Q. L. Yang and Q. Wan, High accurate synchronous clock based on GPS for power grid, Hunan Electric Power. 30 (2010) 23-26.

[2] Z. W. Li, J. Yao and X. Zeng, New method of generating GPS high accuracy synchronous clock based on the digital phase-lock principle, Automation of Electric Power Systems. 33 (2009) 82-86.

[3] B. L. Xu, D. N. Zhang and W. G. Yuan, Design and application of time synchronization system based on multi-sources timing, Journal of Electric Power Science \& Technology. 26 (2011) 25-29.

[4] R. Albert and A.L. Barabbas, Statistical mechanics of complex networks, Rev. Mod. Phys. 74 (2002) 47-97.

[5] S.H. Strogatz, Exploring complex networks, Nature. 410 (2001) 268-276.

[6] H.Y. Sun, Q.L. Zhang and N. Li, Synchronization control of united complex dynamical networks with multi-links, Int. J. Innov. Comput. Inf. Control. 7 (2011) 927-939.

[7] H.Y. Sun, Q.L. Zhang and N. Li, Synchronization of delayed complexdynamical networks via adaptive periodically intermittent control, Control and Decis. 28 (2013) 797-800.

[8] T. Liao and S. Tsai, Adaptive synchronization of chaotic systems and its application to secure communications, Chaos, Solutions \& Fractals. 11 (2000) 1387-1396.

[9] H.Y. Sun, N. Li, D.P. Zhao and Q.L. Zhang, Synchronization of complex networks with coupling delays via adaptive pinning intermittent control, Int. J. Autom. Compute. 10 (2013) 312-318.

[10] N. Li, H.Y. Sun, X. Jing, and Q.L. Zhang, Exponential synchronisation of united complex dynamical networks with multi-links via adaptive periodically intermittent control, IET Control Theory Appl. 7 (2013) 1725-1736.

[11] D.M. Li, J.A. Lu, X.Q. Wu and G.R. Chen, Estimating the ultimate bound and positively invariant set for the Lorenz system and a unified chaotic system, J. Math. Anal. Appl. 323 (2006) 844-853. 